\title{
Perspectives and Outcomes of the Activity of a Reference Laboratory for Brucellosis
}

\author{
Menachem Banai*, Rita Itin and Svetlana Bardenstein \\ Department of Bacteriology, Kimron Veterinary Institute, Bet Dagan, Israel
}

OPEN ACCESS

Edited by:

Xin Ting,

Institute of Animal Sciences

(CAAS), China

Reviewed by:

Hao Dong,

China Animal Disease Control

Center, China

Muhammad Zubair Shabbir, University of Veterinary and Animal

Sciences, Pakistan

*Correspondence:

Menachem Banai

menachemba48@gmail.com

Specialty section:

This article was submitted to Veterinary Infectious Diseases,

a section of the journal

Frontiers in Veterinary Science

Received: 16 October 2017

Accepted: 14 December 2017

Published: 05 January 2018

Citation:

Banai M, Itin R and Bardenstein S

(2018) Perspectives and Outcomes

of the Activity of a Reference

Laboratory for Brucellosis.

Front. Vet. Sci. 4:234.

doi: 10.3389/fvets.2017.00234
One health is an emerging conceptual approach geared to harmonize the activities of the public health, veterinary services, and extension services within a single operative structure. Brucellosis is an important zoonosis worldwide, mostly involving nomadic populations but may often affect transboundary animal management and exotic domesticated animal farming such as camels and buffalo. Here, we provide contemporary knowledge on the disease and its causative agent, a Gram-negative bacteria belonging to the genus Brucella. Further, because of the zoonotic importance, we emphasize the need to assign a national reference laboratory for the disease and discuss how this would integrate into a "One Health" system. Brucella vaccines are live attenuated strains possessing the smooth phenotype, and vaccination, therefore, hampers the ability to maintain a national surveillance program due to concerns regarding the false positive vaccine-induced responses. In order to overcome these failings, we developed a combined approach based on rapid screening of mass numbers of serum samples by the fluorescence polarization assay, a cost-effective and accurate method, and confirmation of the true positive reactors by the complement fixation test, a highly specific method that is less sensitive to vaccine-induced antibodies. We demonstrate how, despite the high vaccination coverage of the small ruminant population in Israel, our results proved to be effective in discriminating between vaccinated and infected animals. The speed and accuracy of the method further justified immediate declaration of $37 \%$ of flocks as cleansed from brucellosis, thus reducing the burden of repeated tests among this population.

Keywords: Brucella, bacteriology, serology, reference laboratory, lipopolysaccharide

\section{INTRODUCTION}

Brucellosis is one of the few severe zoonoses with worldwide distribution (1). The disease is associated with domestic animals, which play important roles in the dairy and meat industries (2) and is disseminated to the human population as a family or tribe cluster infection (3). Awareness of the association of the disease with domestic animals has raised in 1887 when Sir Bruce first identified the organism in pathological human samples, followed by implementation of serological diagnosis and Brucella isolation diagnostic approaches, respectively (4). To date, despite a century of learning, the disease still prevails at high rates in countries of the Latin America, South East Asia, the Middle East, and Persian Gulf (1).

Until the late 1960s, the international community recognized only six Brucella species based upon their unique association with a natural host in which they cause abortion in the 
last trimester of pregnancy (5). Four of these were considered zoonotic: Brucella abortus infecting cattle, but found associated with camelids and other bovids, Brucella melitensis infecting small ruminants but posing risk to bovids and camelids, and Brucella suis and Brucella canis infecting suids and canids, respectively. Two others have been recognized as non-zoonotic Brucella species: Brucella ovis, associated with orchitis and epididymis in rams and Brucella neotomae, infecting wood rats. Interestingly, recent studies have identified two potentially zoonotic sea mammalian species: Brucella ceti and Brucella pinnipedialis, associated with cetacean and pinniped brucellosis, respectively $(6,7)$. Molecular studies further showed that the genomes of the six-species, later conceived "Classical Brucella," were highly homologous, justifying their unification into one single species, $B$. melitensis, upon which their sub-taxon being determined according to morphological and biochemical characteristics (8).

The discovery of two more classical Brucella species isolated from vole and red fox or soil, e.g., Brucella microti, endowed with higher metabolic activity $(9,10)$ and the Brucella papionis baboon-associated strain (11), further extended with the addition of the atypical strains belonging to the more basal lineages, e.g., Brucella inopinata BO1 (12) and BO2 human pathogens, and the red fox Brucella vulpis (13) or the motile frog isolates, has opened the door to widening the genus structure.

Most Brucella species have two chromosomes, of roughly 2.1 and $1.2 \mathrm{Mbp}$, ranging at 57.8\% GC content (14). Brucella species lack common virulence factors such as motility, plasmids, and exotoxins. In contrast, they are equipped with a complete $\operatorname{vir} B$ operon that endows them with an active type IV secretion mechanism (15) and a sheathed flagellum (16) found active in the basal frog lineages (17). The Brucella cell envelope contains lipopolysaccharide (LPS), a molecule shared by all Gram-negative bacteria. Due to a non-canonical structure, its lipid-A conveys a stealthy infection following the development of a poor innate immune response (18). The role Brucella LPS plays in the process of Brucella trafficking into the replicative niche of Brucellacontaining vacuoles is currently debated (19). In contrast, its role as a major humoral stimulating antigen has laid out the structure for the serological diagnosis of brucellosis.

The epidemiology of brucellosis in Israel underwent significant changes throughout the years, affected mainly by the socioeconomic and geopolitical restrains in the region. During the 1950s and 1960s, B. abortus prevailed in the country. The disease was eradicated in 1985 following implementation of a "test and cull" policy combined with a full dose, sub-cutaneous S19 vaccination of replacement females. Later on, the increase in the small ruminant population and market demands eventually led to the emergence of $B$. melitensis in the country. A control program was then instigated in the 1990s based upon Complement Fixation Test (CFT) serological surveys of the adult population and implementation of a combined "test and cull" and live Rev. 1 vaccination program (20). Due to the lack of sustainable financing, the program was ceased in 1997 and the disease re-emerged in the beginning of the 21 st century, leading to a record number of new human cases as well as the infection of a large dairy cattle farm in the southern region of Israel (Negev) (21).

\section{THE CONCEPT OF A REFERENCE LABORATORY FOR BRUCELLOSIS}

The "One health" approach addresses the multi-disciplinary facets of disease complexity involving livestock biosecurity, environmental conditions, veterinary, and medical extension services, as well as farm to fork aspects. Setting up a national reference laboratory complements the "One Health" concept in providing a centralization center with expertise on the standardization of methods corresponding with epidemiology, diagnosis, and human treatment, as well as implementation of prophylaxis and control programs in the livestock population. The laboratory should then focus its activity on strain isolation and typing (gold standard test) and characterization of serological tests that confirm exposure and/or infection among livestock populations and humans, respectively.

\section{Brucella TYPING}

In recent years, a return to a nomen-species structure of genus Brucella has gained support (22). In the laboratory, new isolates are first characterized according to their susceptibility to Fuchsin and Thionin dyes, as well as growth dependence on an enhanced $\mathrm{CO}_{2}$ atmosphere, and $\mathrm{H}_{2} \mathrm{~S}$ and urease production. Staining by the modified Ziehl-Neelsen method is a rapid supplementary technique applied to confirm the disease at point of care sites based upon characteristic cell morphology and staining of Brucella species (23). In past years, Brucella metabolism was analyzed by the old oxygen consumption test, but this test has recently been replaced by a robust microplate metabolome method (24). Use of phage typing has remained supplementary to the methods, but the availability of different sources of phage variants complicate standardization of such analyses (25). Unfortunately, access to brucellaphage seed stocks has become rare and this situation has been worsened by the lack of knowhow on brucellaphage propagation and preparation of master routine test dilutions of phage suspensions emphasizing the need for harmonization and unification of a worldwide Brucella typing and classification system.

\section{Brucella VACCINES}

Only a few Brucella vaccines have been approved for use in the field, all based upon live attenuated strains (26). Live vaccines are superior to killed or acellular vaccines as they survive in the host for a sufficient period of time required to induce a strong immune response by continuously challenging the host immune system. It was estimated that a good vaccine strain must survive for a minimum of $7.9 \pm 1.2$ weeks before clearance of the organisms by the immune system takes place. In contrast, virulent vaccine strains survive longer in the host, ruling out their use as suitable vaccines. The vaccine proficiency of the strain is established as inducing host cellular immune response active in clearance of a challenge strain (27) in comparison to an un-vaccinated control animal $(28,29)$. In contrast, the humoral response plays mainly as a secondary function in conveying protective immunity during early dissemination of the pathogen in the blood (30). 
Unfortunately, antibodies elicited by the vaccine interfere with surveillance and monitoring programs, due to the lack of effective DIVA methods that could distinguish between vaccine and field strain serology. Reference laboratories must play a role in determining vaccine qualities, establishing standard seed stocks and confirming vaccine lot qualities prior to vaccination/usage (20).

Brucella abortus S19/B19 and B. melitensis Rev. 1 have been established as official live attenuated reference vaccine strains for cattle and small ruminants, respectively. In recent years, B. abortus RB51 has been endorsed as a compromised vaccine fulfilling the protective activity against $B$. abortus in cattle without eliciting conflicting smooth antibodies that hamper serological testing (31). Readers are referred to a comprehensive review on the evolutionary adaptation of Brucella species to their hosts and how vaccination may intervene with the emergence of novel Brucella (4). Further reading is recommended regarding vaccine efficacy and risks associated with Rev.1 and S19 implementation at different conditions $(26,32)$.

\section{MOLECULAR TYPING}

Brucella typing and classification can now be achieved by molecular approaches. DNA amplification of a specific sequence by PCR is a basic approach in bacteriology aimed at gene cloning, on the one hand, and gene characterization on the other and is currently widely used in diagnosis (33). AMOS PCR (standing for B. abortus, B. melitensis, B. ovis, and B. suis) was established in the 1990s as a primary method of classification of these species. The method is based on the IS711 sequence inserted at different allelic sites in the chromosomes of the different species, producing characteristic amplified fragments of the target sites. Among B. abortus, AMOS PCR is limited to bvs. 1, 2, and 4, inferring insufficient sensitivity of this method to other B. abortus biovars $(34,35)$. Several other PCR-based molecular methods have been described in the literature culminating to the approval of the Bruce-ladder PCR by the OIE as a recommended approach (36).

Variable number tandem repeats (VNTR) that is also referred to as multiple loci VNTR analysis, is a molecular amplification method of micro- and macro-satellite DNA fragments that depict specific molecular fingerprints associated with an epidemiological strain. The method targets 16 molecular sites on the Brucella chromosome, divided into eight Panel A markers that specifically distinguish between Brucella species and 5 and 3 Panel B hypervariable epidemiological genotypes, respectively (37). Although effective in defining epidemiological linkages among isolates, this method suffers a major flaw by including multiple Brucella biovars within a single genotype.

\section{HIGH-THROUGHPUT MOLECULAR TYPING}

Whole genome sequencing has been instigated in recent years in genomic characterization of bacteria. At the chromosomal level, single-nucleotide polymorphism plays an important tool in identifying phylogenetic linkages. This method has been widely implemented in describing Brucella phylogenetic linkages as well as associating novel strains into genus Brucella. Development of a national bank of a strain collection helps in showing global trends of Brucella spread or clonal evolution for which multilocus-sequence analysis is considered a favored approach (36).

\section{SEROLOGICAL TESTS}

Brucella species cross-react among each other as well as with Yersinia enterocolitica O9 and other Gram-negative bacteria (38). Furthermore, disease first progresses with the development of IgM antibodies and further succeeds by the development of IgG antibodies during the chronic state or alongside pathogen persistence in the animal (39). Finally, antibody isotypes might vary upon the challenge dose, the site of infection, and the capacity to establish bacteremia or develop a limited local infection in a specific organ or tissue, as well as antibiotic treatment. Therefore, these factors must be taken into account when designing a serological approach in the laboratory.

A reciprocal correlation exists between a test's sensitivity and its specificity. Sensitivity is increased at the expense of increased background noise, therefore, reducing specificity. In contrast, specificity is increased by reducing background noise and cross reactivity with heterologous antibodies at the expense of reduced sensitivity. IgM antibodies are pentamers, which promote the development of a net between antibodies and cells, thus causing agglutination in the tube [serum agglutination test (SAT)]. In contrast, IgG antibodies interact with the host's complement fixing system. Such a function forms the basis of the CFT, a lytic readout response against targeted antigens. Because IgM and IgG antibodies rise at different stages of the disease, agglutination and CFT complement each other in diagnosis, the first highlighting on acute infection whereas the latter indicates a chronic persisting infection, respectively. Thus, CFT provides a higher predictive value than SAT and, as such, has been affirmed by OIE as the prescribed method for animal trade between countries.

The enzyme-linked immunosorbent assay (ELISA) and fluorescence polarization assay (FPA) are two high-throughput serological techniques aimed at detecting serum antibodies (analyte) in a given sample. Because the two systems employ different underlying principles, their performance by sensitivity and specificity may vary. Two ELISA methods have been validated, indirect ELISA that measures binding of secondary antibodies to a primary antibody isotype bound onto the Brucella LPS antigen, and competitive ELISA, which measures the competitive binding between anti-Brucella LPS monoclonal antibodies and host's antibody onto the same reactive site. Both ELISA methods suffer from having non-specific binding of conjugate to background substrates and as such, their reading signal is increased. To avoid background noise, blocking and washing steps are introduced as intermediated steps in the method, thus reducing analyte concentration in the system and reducing the test's sensitivity.

Unlike ELISA, FPA measures antibody (analyte) binding to a soluble antigen by a homogenous measurement method without interventional steps such as blocking and washings. Because of the principle of homogeneity, this method measures the interaction of all participating antibody isotypes in the sample with 
the soluble antigen, establishing the highest final reading. It is considered, therefore, highly sensitive. Test specificity has been similarly achieved due to using the soluble Brucella O-PS antigen, which omits Lipid A and core LPS epitopes in the reaction mixture. The method thus achieves a maximum performance value of close to 200\%, surpassing ELISA as the best performing technique (40).

Plate agglutination methods, such as Rose Bengal Test (RBT), resemble FPA as homogenous methods. Unlike FPA, RBT is performed manually, limiting the number of samples a laboratory can process per day. RBT is considered, therefore, a screening approach surpassed by the high-throughput ELISA and FPA methods, however, because of its low price, some laboratories rather use this test as an alternative test to CFT.

The establishment of an international standard serum, which aimed to internationally harmonize test results, was considered by the OIE, leading to development of the second International standard anti-Brucella abortus Serum (second ISABS) arbitrarily assigned with 1,000 IU for SAT and CFT tests, respectively (23). Local serum standards could thus be established in different countries, allowing for the first time, the comparison of serological tests among different countries. A similar serum standard against $B$. melitensis infection in the small ruminant population has recently been described (41). Nonetheless, neither standard sera directly correlate with the diagnosis of human brucellosis.

\section{DECISION-MAKING}

Small ruminant population in Israel includes intensively managed flocks grown in rural places for dairy and meat

TABLE 1 | Calculation of the agreement between fluorescence polarization assay (FPA) and Complement Fixation Test (CFT) observations by the Cohen's Kappa test.

\begin{tabular}{lccr}
\hline & FPA positive & FPA susceptible & Total \\
\hline CFT positive & 97 & 10 & 107 \\
CFT negative & 269 & 725 & 994 \\
Total & 366 & 735 & 1,101 \\
\hline
\end{tabular}

Number of observed agreements: 822 (74.66\% of the observations).

Number of agreements expected by chance: 699.1 (63.50\% of the observations). Kappa $=0.306$.

SE of kappa $=0.027$

95\% confidence interval: from 0.253 to 0.358 .

The strength of agreement is considered to be "fair."

TABLE 2 | Comparison of numbers of responders between fluorescence polarization assay (FPA) and Complement Fixation Test (CFT) among serum samples randomly taken at early stages of the 2016 campaign.

\begin{tabular}{lccc} 
& FPA positive & FPA suspect & FPAn \\
\hline CFTp & 3,066 & 3,179 & 37,511 \\
CFT & $2,255(36.1 \%)$ & \\
\end{tabular}

P, positive; N, negative; S, suspected. production and extensively managed open flocks often pasteurizing on agricultural lands at southern Israel (Negev), and less at the northern part of the country. Human brucellosis is mostly associated with the nomadic animals due to close contacts with the animals. Following the 1990s' campaign (20), implementation of a full dose, ocular Rev. 1 vaccination of the young replacement females was enforced in the country. In spite of the vaccination program, due to cessation of the national control program, a new burst of human cases rose, most occurring in the Negev, leading to a public outcry to instigate a new national campaign.

The new program targeted the Negev, covering about 250 pasteurizing flocks (some 15,000 animals) in close proximity to dairy cattle herds, and others of risk to humans, including more than 1,000 flocks (over 250,000 heads) respectively.

In order to overcome DIVA concerns, we chose FPA as a screening method (being rapid and highly sensitive), and CFT [omitting most vaccine reacting individuals from the population (42)] as a confirmative method.

\section{RESULTS AND CONCLUSION}

For statistical purposes, we chose sampling data that were available from the second cycle of the brucellosis campaign. At this stage, we expected most of the population to be tested negative by FPA as most infected animals have already been excluded from the population. Other FPA results could have been distributed between FPA positive (FPAp) and FPA suspect (FPAs) reactors, respectively. Among these reactors, we anticipated that FPAs reactors would belong to the vaccinated population expected to be found CFT negative (CFTn). We chose a free "QuickCalcs" calculator, which compares agreement between two methods in terms of a Kappa value, based upon the number of selected categories in the system. Comparison was conducted upon the observations obtained from the two population categories, FPAp:CFTp and FPAs:CFTn, respectively. As shown in Table 1, a total of 4,684 animals were tested by the FPA method revealing $1,101(23.5 \%)$ of FPAp and FPAs responders in total that further were distributed within groups as follows: FPAp:CFTp $(97,8.8 \%)$, FPAs:CFTp (10, 0.9\%), FPAp:CFTn (269, 24.4\%), and FPAs:CFTn $(725,65.8 \%)$, respectively. As shown in Table 1, the inter-rater qualitative agreement by Cohen's Kapp test indicated fair agreement between FPA and CFT tests, with Kappa coefficient of 0.306 (95\% CI 0.253-0.358).

As seen in Table 2, we tested 43,756 animals representing 760 flocks during the first cycle of the campaign. FPAs $(3,179)$ and FPAp $(3,066)$ comprised approximately $14.3 \%$ of the population. At the flock category, a total of $482(63.2 \%)$ flocks were suspect/positive by FPA, 203 (26.7\%) comprised true CFTp and 279 (36.7\%) were CFTn, respectively, allowing omission of the latter group from future monitoring and thus reducing the burden on the laboratory. This highlights on the advantage of using FPA on a wide scale surveillance campaign in a population with a high vaccine coverage, first as a screening approach, using CFT as a confirmation method, and then, 
as a sole method during the finalization of the eradication campaign. In conclusion, our work confirms the feasibility of using FPA in screening and eradication of vaccinated flocks in other places worldwide.

\section{ETHICS STATEMENT}

The study was exempt from one or more of the above requirements because it was conducted for the purpose of the control/ eradication of brucellosis during a national campaign.

\section{REFERENCES}

1. Pappas G, Papadimitriou P, Akritidis N, Christou L, Tsianos EV. The new global map of human brucellosis. Lancet Infect Dis (2006) 6:91-9. doi:10.1016/ S1473-3099(06)70382-6

2. Corbel MJ. Epidemiology. In: Corbel MJ, Elberg SS, Cosivi O, editors. Brucellosis in Humans and Animals, WHO/CDS/EPR/2006.7. Geneva 27, Switzerland: World Health Organization, Food and Agriculture Organization of the United Nations, World Organization for Animal Health, WHO Publications (2006). pp. 1-89.

3. Fosgate GT, Carpenter TE, Chomel BB, Case JT, DeBess EE, Reilly KF. Time-space clustering of human brucellosis, California, 1973-1992. Emerg Infect Dis (2002) 8:672-8. doi:10.3201/eid0807.010351

4. Moreno E. Retrospective and perspectives on zoonotic brucellosis. Front Microbiol (2014) 5:213. doi:10.3389/fmicb.2014.00213

5. Aparicio ED. Epidemiology of brucellosis in domestic animals caused by Brucella melitensis, Brucella suis and Brucella abortus. Rev Sci Tech (2013) 32(43-51):53-60. doi:10.20506/rst.32.1.2187

6. Guzmán-Verri C, González-Barrientos R, Hernández-Mora G, Morales JA, Baquero-Calvo E, Chaves-Olarte E, et al. Brucella ceti and brucellosis in cetaceans. Front Cell Infect Microbiol (2012) 2:3. doi:10.3389/fcimb.2012.00003

7. Nymo IH, Tryland M, Godfroid JA. Review of Brucella infection in marine mammals, with special emphasis on Brucella pinnipedialis in the hooded seal (Cystophora cristata). Vet Res (2011) 42:93. doi:10.1186/1297-9716-42-93

8. Verger J-M, Grimont F, Grimont PAD, Grayon M. Brucella, a monospecific genus as shown by deoxyribonucleic acid hybridization. Int J Syst Bacteriol (1985) 35:292-5. doi:10.1099/00207713-35-3-292

9. Scholz HC, Hubalek Z, Nesvadbova J, Tomaso H, Vergnaud G, Le Flèche P, et al. Isolation of Brucella microti from soil. Emerg Infect Dis (2008) 14:1316-7. doi:10.3201/eid1408.080286

10. Scholz HC, Hubalek Z, Sedlácek I, Vergnaud G, Tomaso H, Al Dahouk S, et al. Brucella microti sp. nov., isolated from the common vole Microtus arvalis. Int J Syst Evol Microbiol (2008) 58(Pt 2):375-82. doi:10.1099/ijs.0.65356-0

11. Whatmore AM, Davison N, Cloeckaert A, Al Dahouk S, Zygmunt MS, Brew SD, et al. Brucella papionis sp. nov., isolated from baboons (Papio spp.). Int J Syst Evol Microbiol (2014) 64:4120-8. doi:10.1099/ijs.0.065482-0

12. Scholz HC, Nöckler K, Göllner C, Bahn P, Vergnaud G, Tomaso H, et al. Brucella inopinata sp. nov., isolated from a breast implant infection. Int J Syst Evol Microbiol (2010) 60:801-8. doi:10.1099/ijs.0.011148-0

13. Scholz HC, Revilla-Fernández S, Al Dahouk S, Hammerl JA, Zygmunt MS, Cloeckaert A, et al. Brucella vulpis sp. nov., isolated from mandibular lymph nodes of red foxes (Vulpes vulpes). Int J Syst Evol Microbiol (2016) 66:2090-8. doi:10.1099/ijsem.0.000998

14. DelVecchio VG, Kapatral V, Redkar RJ, Patra G, Mujer C, Los T, et al. The genome sequence of the facultative intracellular pathogen Brucella melitensis. Proc Natl Acad Sci U S A (2002) 99:443-8. doi:10.1073/pnas.221575398

15. Delrue R-M, Deschamps C, Léonard S, Nijskens C, Danese I, Schaus J-M, et al. A quorum-sensing regulator controls expression of both the type IV secretion system and the flagellar apparatus of Brucella melitensis. Cell Microbiol (2005) 7:1151-61. doi:10.1111/j.1462-5822.2005.00543.x

16. Fretin D, Fauconnier A, Kölher S, Halling S, Léonard S, Nijskens C, et al. The sheathed flagellum of Brucella melitensis is involved in persistence in a murine model of infection. Cell Microbiol (2005) 7:687-98. doi:10.1111/j.1462-5822.2005.00502.x

\section{AUTHOR CONTRIBUTIONS}

$\mathrm{MB}$ conceived the project and wrote the manuscript. SB produced the data and RI assisted in performing the tests and data organization.

\section{FUNDING}

Research was supported by Grant No. 845-0289-13 from Chief scientist, Ministry of Agriculture. We thank our Israeli colleagues at the Veterinary District Office, Beer Sheva, and support provided by the $\mathrm{CVO}$ and Israeli veterinary services.

17. Al Dahouk S, Köhler S, Occhialini A, de Bagüés MPJ, Hammerl JA, Eisenberg $\mathrm{T}$, et al. Brucella spp. of amphibians comprise genomically diverse motile strains competent for replication in macrophages and survival in mammalian hosts. Sci Rep (2017) 7:44420. doi:10.1038/srep44420

18. Barquero-Calvo E, Chaves-Olarte E, Weiss DS, Guzmán-Verri C, ChacónDíaz C, Rucavado A, et al. Brucella abortus uses a stealthy strategy to avoid activation of the innate immune system during the onset of infection. PLoS One (2007) 18:2. doi:10.1371/journal.pone.0000631

19. von Bargen K, Gorvel J-P, Salcedo SP. Internal affairs: investigating the Brucella intracellular lifestyle. FEMS Microbiol Rev (2012) 36:533-62. doi:10.1111/j.1574-6976.2012.00334.x

20. Banai M. Control of small ruminant brucellosis by use of Brucella melitensis Rev. 1 vaccine: laboratory aspects and field applications. Vet Microbiol (2002) 90:497-519. doi:10.1016/S0378-1135(02)00231-6

21. van Straten M, Bardenstein S, Keningswald G, Banai M. Brucella abortus S19 vaccine protects dairy cattle against natural infection with Brucella melitensis. Vaccine (2016) 34:5837-9. doi:10.1016/j.vaccine.2016.10.011

22. Osterman B, Moriyón I. International committee on systematics of prokaryotes subcommittee on the taxonomy of Brucella. Minutes of the meeting, 17 September 2003, Pamplona, Spain. Int J Syst Evol Microbiol (2006) 56:1173-5. doi:10.1099/ijs.0.64349-0

23. Alton GG, Jones LM, Angus RD, Verger J-M. Techniques for the Brucellosis Laboratory. Paris: INRA Publications (1988).

24. Al Dahouk S, Scholz HC, Tomaso H, Bahn P, Göllner C, Karges W, et al. Differential phenotyping of Brucella species using a newly developed semi-automated metabolic system. BMC Microbiol (2010) 10:269. doi:10.1186/1471-2180-10-269

25. Hammerl JA, Göllner C, Jäckel C, Scholz HC, Nöckler K, Reetz J, et al. Genetic diversity of Brucella reference and non-reference phages and its impact on Brucella-typing. Front Microbiol (2017) 8:408. doi:10.3389/ fmicb.2017.00408

26. World Organization for Animal Health. Chapter 2.1.4 brucellosis (Brucella abortus, B. melitensis, B. suis) (infection with B. abortus, B. melitensis and B. suis). Manual of Diagnostic Tests and Vaccines for Terrestrial Animals. Paris: OIE (2017). Available from: http://www.oie.int/en/international-standard-setting/ terrestrial-manual/access-online/

27. Oliveira SC, Splitter GA. CD8+ Type 1 CD44hi CD45 RBlo T lymphocytes control intracellular Brucella abortus infection as demonstrated in major histocompatibility complex class I- and class II-deficient mice. Eur J Immunol (1995) 25:2551-7. doi:10.1002/eji.1830250922

28. Garin-Bastuji B. The quality assurance of living Brucella-vaccines, the French experience with the Rev. 1 vaccine. In: Garin -Bastuji B, Benkirane A, editors. FAO/WHO/OIE Round Table on the Use of Rev. 1 Vaccine in Small Ruminants and Cattle. Alfort, France: CNEVA (1995). p. 45-50.

29. Grilló M-J, Blasco JM, Gorvel JP, Moriyón I, Moreno E. What have we learned from brucellosis in the mouse model? Vet Res (2012) 43:29. doi:10.1186/1297-9716-43-29

30. Vitry MA, Hanot Mambres D, De Trez C, Akira S, Ryffel B, Letesson JJ, et al. Humoral immunity and CD4+ Th1 cells are both necessary for a fully protective immune response upon secondary infection with Brucella melitensis. J Immunol (2014) 192:3740-52. doi:10.4049/jimmunol.1302561

31. Dorneles EMS, Sriranganathan N, Lage AP. Recent advances in Brucella abortus vaccines. Vet Res (2015) 46:76. doi:10.1186/s13567-015-0199-7 
32. Blasco JM. A review of the use of B. melitensis Rev. 1 vaccine in adult sheep and goats. Prev Vet Med (1997) 31(3-4):275-83. doi:10.1016/S0167-5877(96) 01110-5

33. Bricker BJ. PCR as a diagnostic tool for brucellosis. Vet Microbiol (2002) 90:435-46. doi:10.1016/S0378-1135(02)00228-6

34. Bricker BJ, Halling SM. Differentiation of Brucella abortus bv. 1, 2, and 4, Brucella melitensis, Brucella ovis, and Brucella suis bv. 1 by PCR. JClin Microbiol (1994) 32:2660-6.

35. Ewalt DR, Bricker BJ. Validation of the abbreviated Brucella AMOS PCR as a rapid screening method for differentiation of Brucella abortus field strain isolates and the vaccine strains, 19 and RB51. J Clin Microbiol (2000) 38:3085-6.

36. Scholz HC, Vergnaud G. Molecular characterisation of Brucella species. Rev Sci Tech (2013) 32:149-62. doi:10.20506/rst.32.1.2189

37. Le Flèche P, Jacques I, Grayon M, Al Dahouk S, Bouchon P, Denoeud F, et al. Evaluation and selection of tandem repeat loci for a Brucella MLVA typing assay. BMC Microbiol (2006) 6:9. doi:10.1186/1471-2180-6-9

38. Muñoz M, Marín CM, Monreal D, González D, Garin-Bastuji B, Díaz R, et al. Efficacy of several serological tests and antigens for diagnosis of bovine brucellosis in the presence of false-positive serological results due to Yersinia enterocolitica O:9. Clin Diagn Lab Immunol (2005) 12:141-51. doi:10.1128/ CDLI.12.1.141-151.2005

39. Godfroid J, Nielsen K, Saegerman C. Diagnosis of brucellosis in livestock and wildlife. Croat Med J (2010) 51:296-305. doi:10.3325/cmj.2010.51.296
40. Gall D, Nielsen K. Serological diagnosis of bovine brucellosis: a review of test performance and cost comparison. Rev Sci Tech (2004) 23:989-1002. doi:10.20506/rst.23.3.1545

41. McGiven J, Taylor A, Duncombe L, Sayers R, Albert D, Banai M, et al. The first international standard anti-Brucella melitensis serum. Rev Sci Tech (2011) 30:809-19. doi:10.20506/rst.30.3.2076

42. Worthington RW, Mülders MSG, McFarlene IS, Becker D. Serological titers following vaccination of sheep and goats with Brucella melitensis Rev. 1 vaccine. Onderstepoort J Vet Res (1973) 40:1-6.

Conflict of Interest Statement: The authors declare that the research was conducted in the absence of any commercial or financial relationships that could be construed as a potential conflict of interest.

The handling editor is currently co-organizing a research topic with one of the authors $\mathrm{MB}$ and confirms the absence of any other collaboration.

Copyright (c) 2018 Banai, Itin and Bardenstein. This is an open-access article distributed under the terms of the Creative Commons Attribution License (CC BY). The use, distribution or reproduction in other forums is permitted, provided the original author(s) or licensor are credited and that the original publication in this journal is cited, in accordance with accepted academic practice. No use, distribution or reproduction is permitted which does not comply with these terms. 